\title{
Using Conference Servers for SIP-based Vertical Handoff between IP and Cellular Networks
}

\author{
Arpit Mehta, Andrea G. Forte, Henning Schulzrinne \\ Department of Computer Science \\ Columbia University, New York \\ am2866@columbia.edu, \{andreaf, hgs\}@cs.columbia.edu
}

\begin{abstract}
The Session Initiation Protocol (SIP) is the preferred signaling protocol for creating and terminating multimedia sessions in an IP network. Also, voice over IP is becoming the favored means of voice communication mainly because of its low cost when compared to voice communication over traditional networks like cellular and PSTN. Therefore, seamless transfer of calls or handoff between the traditional and IP networks becomes important.

We describe a technique to achieve seamless mid-call handoffs in a scenario where a handoff occurs between the cellular network and the IP network. The technique we describe makes use of a network element acting as an ISDN gateway, SIP conference server and SIP proxy server. We have measured handoff delays from IP to ISDN network and vice-versa. When using the proposed soft handoff technique, these delays are $0.10 \mathrm{~s}$ and $0.02 \mathrm{~s}$, respectively. Finally, we share the results and some of the challenges we faced while implementing the overall mechanism on a dual-mode handset.
\end{abstract}

\section{Categories and Subject Descriptors}

C.2.1 [Computer-Communication Networks]: Network Architecture and Design-Wireless communication

\section{General Terms}

Algorithms, Design

\section{Keywords}

FMC, conference, SIP, handoff, vertical

\section{INTRODUCTION}

Fixed - Mobile Convergence (FMC) [1] is defined as the integration of wired and wireless networks and services allowing users to seamlessly switch between wired and wireless networks. To achieve seamless switching, handoff from one network to another is required. A handoff that occurs between different networks of different technologies is known as vertical handoff. Because of

Permission to make digital or hard copies of all or part of this work for personal or classroom use is granted without fee provided that copies are not made or distributed for profit or commercial advantage and that copies bear this notice and the full citation on the first page. To copy otherwise, to republish, to post on servers or to redistribute to lists, requires prior specific permission and/or a fee.

MobiWac'08, October 30-31, 2008, Vancouver, BC, Canada.

Copyright 2008 ACM 978-1-60558-055-5/08/10 ...\$5.00.

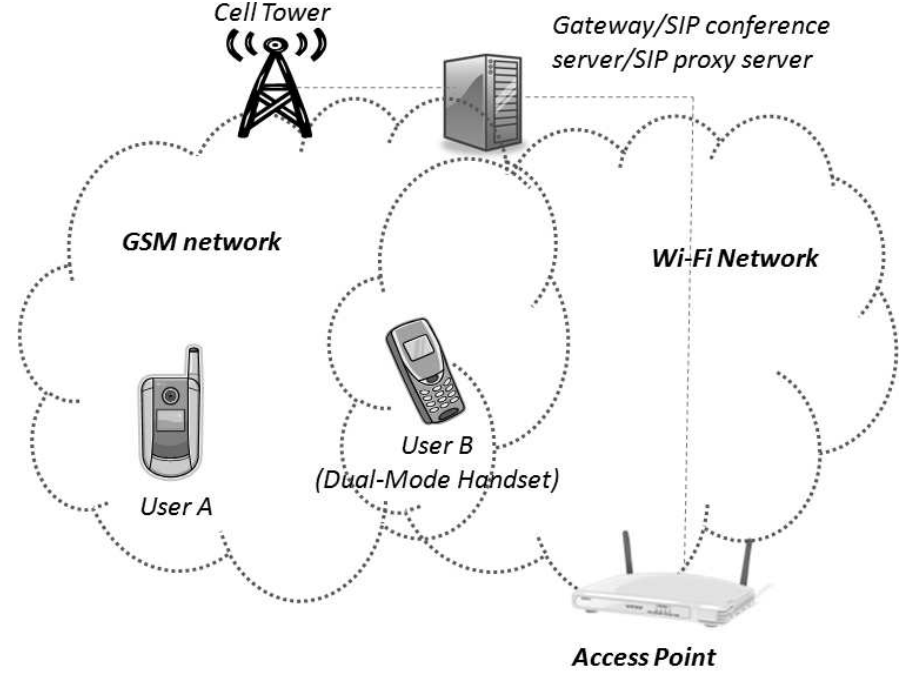

Figure 1: Architecture overview

the growing importance of FMC, 3GPP has approved an item [2] on this service called Voice Call Continuity (VCC). VCC provides session continuity across networks such as $2 \mathrm{G}$ to $2 \mathrm{G}$ networks, $3 \mathrm{G}$ to $3 \mathrm{G}$ networks, $2 \mathrm{G}$ GSM and $3 \mathrm{G}$ UMTS networks and also between cellular network and wireless LANs (WLANs). In the case of WLAN handoffs, the location of the WLAN Access Point (AP) should also be covered by the cellular service provider.

Session mobility is another approach, which allows the user to transfer an ongoing multimedia session from one device to another device. Session mobility is achieved at the application layer and is independent of mobility at lower layers. Furthermore, with session mobility, the session could be transferred to multiple devices of varying specifications and it is not necessarily seamless [3].

Our design achieves seamless vertical handoffs between cellular technologies like GSM or CDMA and IP-based networks. We make use of a network element which acts as a PBX, a conference server and a SIP proxy server. One of our goals for the design was to minimize changes in the standard network components and the handset of the end user. In our design, only the users who wants to achieve seamless mobility have to modify their handsets to support our technique. No changes are required on the handset of the user mounting a single network interface.

The proposed technique makes use of a SIP conference server, SIP proxy server, ISDN gateway and call forwarding. Figure 1 shows the various network elements. There are two users, A and B. A is on a cellular network while B has a dual mode handset 
with both cellular and IP connectivity. Whenever user B makes or receives a call, the call is routed to the SIP conference server, a conference for that call is created and the call is added to the conference. For example, when user B receives a call from user A, the call is forwarded by the network of user B to the SIP conference server and user $\mathrm{A}$ is added to a conference. At this point, the conference server makes a call back to user B and user B is added to the same conference. Just before the handoff occurs, the second network interface of user $B$ is added to the same conference. For a very short duration, user A and both interfaces of user B are joined in the same conference. Once the handoff has completed, the first interface of user B is not used any longer and is removed from the conference. Now user A and user B are in the same conference using one interface each. Similar is the case when user B makes a call to user A. Again, a new conference is dynamically created with only user B initially in that conference. User A is added to the same conference after a call is made to user A. A handoff takes place in the same way as in the previous scenario.

In our implementation, we have used Asterisk [4], an open-source PBX, as a SIP proxy server, an ISDN gateway and a SIP conference server. For the conference part, we have used an application called MeetMe, which is the conference component in Asterisk.

The rest of the paper is organized as follows. In Section 2, we introduce related work, Section 3 gives a description of call forwarding and Section 4 describes the various possible user scenarios. In Section 5 we describe in detail how the handoff is achieved. Section 6 presents the experimental results of our proposed mechanism, Section 7 describes some of the implementation details and Section 8 presents the problems we faced in developing a solution for our dual-mode client. Finally, Section 9 concludes the paper.

\section{RELATED WORK}

A large number of vertical handoff techniques exist in literature, however most of them do not achieve seamless handoffs between $2 \mathrm{G}$ cellular networks and IP networks. Here, we briefly describe some of the existing techniques which we feel are relevant to our problem.

In [3], Shacham et al have introduced an approach for session mobility using SIP. They have defined a framework which allows a device to discover other available devices and transfer active multimedia sessions between these devices.

In [5], the authors discuss the aspects that must be addressed for seamless mobility between IP Multimedia Subsystem (IMS) networks and any other wireless network. Call delivery, roaming, handoff, and feature parity across disparate networks are important issues that must be addressed to provide users with benefits from both networks. Their solution is based on IMS networks and they describe a high level architecture necessary for seamless mobility. However, they have not implemented it and therefore are not able to study and verify the performance of their approach.

A SIP-based mobility architecture for soft handoff for IP-centric wireless networks has been proposed in [6]. The mobile client performing the handoff has two IP interfaces. The handoff is initiated by the mobile client by sending an INVITE message with the Join header to a SIP proxy server. During the handoff the mobile client sends and receives packets through both interfaces. When packets reach the mobile client through the new interface, a re-INVITE message is sent with the IP address of the newly active interface and corresponding contact information. When call renegotiation is complete, a BYE message is sent to terminate the leg of the call going through the old interface. Their handoff procedure ensures no packet loss and acceptable delay jitter but only for handoffs between IP-based networks.

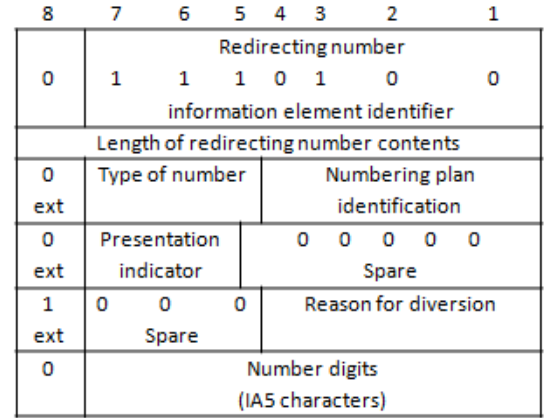

Figure 2: ISDN Redirecting Number Information Element [10]

In [7], the authors propose a technique to provide seamless voice service using SIP in an heterogeneous environment, containing different radio technologies. They take advantage of Session Border Controllers (SBC) to deal with Network Address Translation (NAT) traversal. The support for NATs combined with mobility and handoff is important as many wireless access networks currently use private IP addresses and are connected through NATs. In their design, the mobile client initiates the handoff by sending a REGISTER message to the SBC using the new network interface. When the REGISTER message is sent, the mobile client starts duplicating and sending media packets from both the old and the new interface. At the other end the SBC forwards the media packets received from the new interface, to the correspondent node. When the mobile client receives a 200 OK from the SBC, it stops duplicating the packets on the two interfaces thus concluding the handoff. The entire handoff process is handled by the mobile client and the SBC, with the correspondent node being completely unaware of it. Their technique relies on the SBC being deployed in the network and their handoff technique works only between IP-based technologies. Non-IP-based networks are not considered.

To the best of our knowledge, the only conference-based approach has been proposed in [8]. Unfortunately, it is rather unclear how the authors have achieved seamless handoffs since the paper only discusses the high level aspects of the handoff. It does not describe how and when exactly the handoff is done. Also, no results for the handoff mechanism are provided.

Other approaches such as [9] have proposed an architecture integrating two major IP-based mobility management architectures, Mobile IP and SIP, for advanced handoff management. This, once again, requires changes in the existing protocols and architecture.

We propose a technique that makes use of an open source PBX with SIP signaling and off-the-shelf equipment. Our approach has the advantage of not introducing changes to the existing network architecture and protocols, while still achieving seamless mobility. In particular, the proposed technique works well also for non-IPbased networks such as GSM cellular networks.

\section{CALL FORWARDING IN GSM NETWORKS}

As we discussed earlier, call forwarding plays an important role in our design. The call forwarding supplementary service enables the user to have the network redirect the calls which are addressed to the user's number to another number [12]. The diverted-to user is the user to which the call has been forwarded or diverted to. The diverted-from user is the user the call has been forwarded or diverted from. 


\begin{tabular}{|c|c|c|c|c|}
\hline & \multicolumn{4}{|c|}{ Redirecting party $\mathrm{BCD}$ number IEI } \\
\hline \multicolumn{5}{|c|}{$\begin{array}{l}\text { Length of redirecting party } \mathrm{BCD} \text { number } \\
\text { contents }\end{array}$} \\
\hline $\begin{array}{l}0 / 1 \\
\text { ext }\end{array}$ & \multicolumn{2}{|c|}{$\begin{array}{l}\text { type of } \\
\text { number }\end{array}$} & \multicolumn{2}{|c|}{$\begin{array}{l}\text { Numbering plan } \\
\text { identification }\end{array}$} \\
\hline $\begin{array}{c}1 \\
\text { ext }\end{array}$ & $\begin{array}{c}\text { presentat } \\
\text { indicator }\end{array}$ & 0 & $\begin{array}{l}0 \\
\text { spare }\end{array}$ & $\begin{array}{l}\text { Screening } \\
\text { indicator }\end{array}$ \\
\hline \multicolumn{3}{|c|}{ Number digit 2} & \multicolumn{2}{|c|}{ Number digit 1} \\
\hline \multicolumn{3}{|c|}{ Number digit 4} & \multicolumn{2}{|c|}{ Number digit 3} \\
\hline & & & & \\
\hline
\end{tabular}

Figure 3: GSM Redirecting Party BCD Number Information Element [11]

Table 1: Handoff scenarios

\begin{tabular}{|l|r|r|r|r|}
\hline Scenario & Caller & Callee & handoff from & handoff to \\
\hline 1 & A & B & GSM & IP \\
\hline 2 & A & B & IP & GSM \\
\hline 3 & B & A & IP & GSM \\
\hline 4 & B & A & GSM & IP \\
\hline
\end{tabular}

Fig. 2 shows the format of the Redirecting Number information element in ISDN. The purpose of the Redirecting Number information element [10] is for the diverted-to user to identify the ISDN number from which diversion was invoked. Here, Number Digits represents the user's number from which the call has been forwarded.

Fig. 3 shows the format of the Redirecting Party BCD Number information element in GSM. The purpose of the Redirecting Party BCD Number information element [11] is to identify the redirecting party. The Redirecting Party BCD Number element contains the number of the redirecting party. Number digit 1 , Number digit 2 and so on contain the redirecting user's number.

The Call Forwarding Unconditional (CFU) service causes blind forwarding of a call to a particular number. In our scenario, $\mathrm{CFU}$ creates a looping problem. In particular, when user A calls user $\mathrm{B}$, the call is automatically forwarded because of CFU, by user B's cellular operator to the gateway. When the call reaches the gateway, it calls user B but since CFU is used by B, the call initiated by the gateway gets forwarded to the gateway again, thus creating a loop. Ideally, we would use a selective call forwarding where the network of the user can selectively choose not to forward calls from a particular number. In our scenario, the network of the user would not forward any call initiated by the gateway.

In our experiments, however, since selective call forwarding is not available, we use call forwarding on busy (CFB). When using $\mathrm{CFB}$, a call is initially routed to the callee and forwarded to a different number only if the callee's device returns a busy signal. In our experiments we trigger a busy signal on the callee's device and therefore call forwarding, every time a call is received from any number but the gateway's. In doing so, the looping problem is avoided.

\section{USER SCENARIOS}

The design of the proposed handoff technique is based on forwarding the incoming call to an ISDN gateway that acts also as a SIP conference server and SIP proxy server. Such server/gateway, for example, could be provided by the company the user works for.
We assume the non-IP interface to be a 2G GSM interface and the IP interface to be an IEEE 802.11 interface. The proposed mechanism however, could work with any type of $\{$ non-IP, IP $\}$ interface pairs. All four possible handoff scenarios are shown in Table 1 and described in the following sections.

\subsection{Scenario 1: User A's cellular interface calls user B's cellular interface}

In this scenario, user A makes a call to user B on its GSM network. This scenario is depicted in Fig. 4.

1. User A calls user B on the GSM network.

2. User B's network forwards the call initiated by user A to the ISDN PRI gateway using CFB.

3. The ISDN PRI gateway calls back user B on the GSM interface. Now the call from A to B is divided into two segments, one from user A to the gateway and the other from the gateway to user B.

4. If user B moves to a location where there is an 802.11 network available, handoff to the IP network occurs (see Section $5)$.

\subsection{Scenario 2: User A's cellular interface calls user B's IP interface}

In this scenario, user A calls user B when user B is connected to an 802.11 network. It is different from the previous scenario as in this case the gateway makes a call to user B on the IP interface and not on the GSM interface. This scenario is depicted in Fig. 5.

1. User A calls user B on the GSM network.

2. The call gets forwarded by user B's GSM network to the ISDN PRI gateway using CFB.

3. User B is using the 802.11 network, therefore the ISDN PRI gateway calls user B on the IP interface. Now the call from A to $\mathrm{B}$ is divided into two segments, one from user A to the the gateway on the cellular network and the other from the gateway to user B on the IP network.

4. If user B moves to a location where there is no 802.11 network available, handoff to the cellular network occurs (see Section 5).

\subsection{Scenario 3: User B's IP interface calls user A's cellular interface}

In this scenario, user B is on the IP network and places a call to user A. This scenario is depicted in Fig. 6.

1. User B calls user A via its IP interface. This call gets routed via the ISDN PRI gateway which also acts as a SIP proxy.

2. The gateway then calls user A on the cellular network. At this point $\mathrm{A}$ and $\mathrm{B}$ are both connected.

3. When user B moves out of the 802.11 enabled region, handoff to the cellular network occurs and the users get connected again with user B using the GSM network, that is, handoff to the cellular network occurs. 


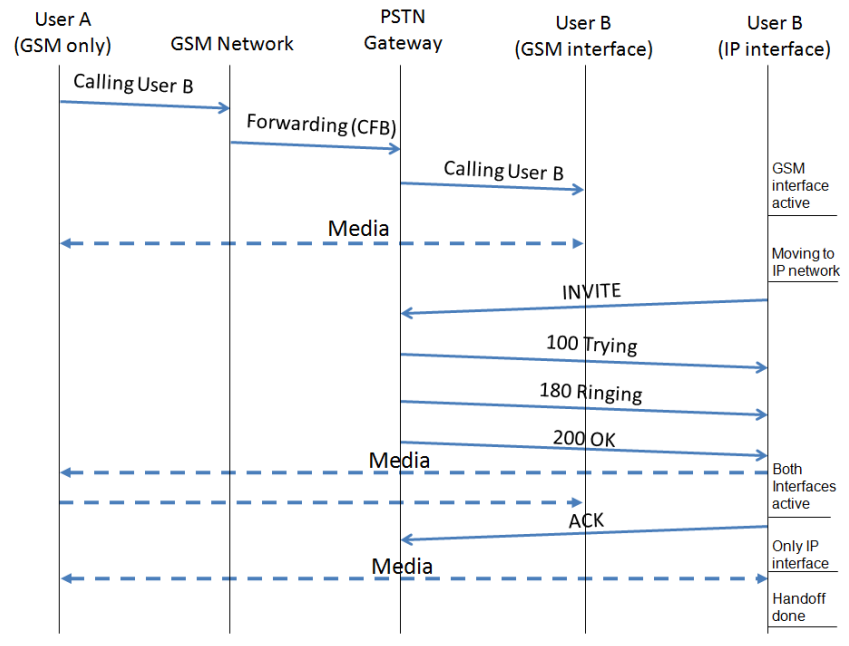

Figure 4: Handoff from the GSM network to the IP network when user $B$ receives a call from user $A$

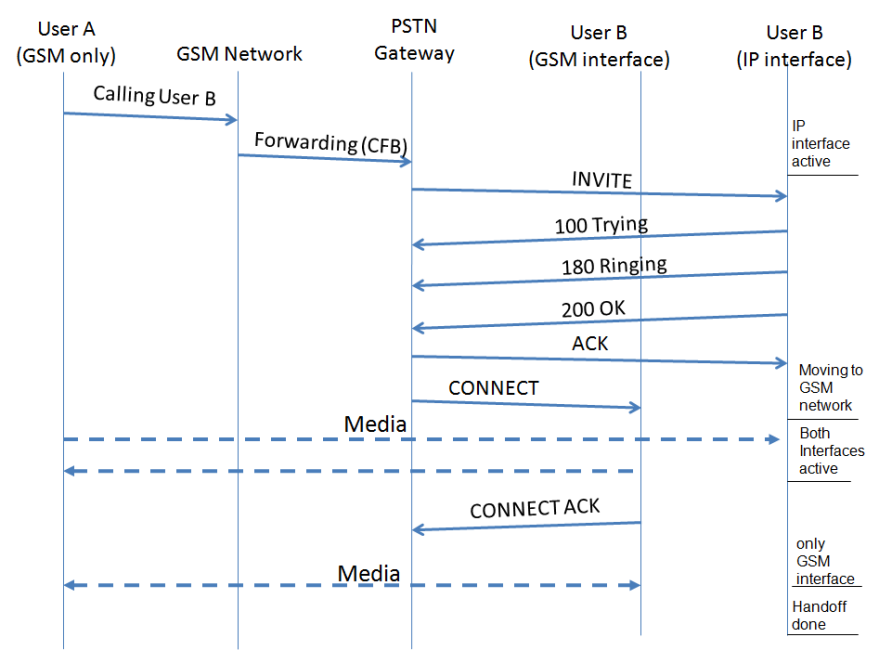

Figure 5: Handoff from the IP network to the GSM network when the user $B$ receives a call from user $A$

\subsection{Scenario 4: User B's cellular interface calls user A's cellular interface}

In this scenario, user B makes a call using its GSM interface to user A. This scenario is depicted in Fig. 7.

1. User B makes a call to user A via its GSM interface. The call is automatically routed to the gateway first.

2. The gateway then makes an ISDN call to user A. User A and user B are now connected.

3. If user B reaches an 802.11 enabled region, handoff occurs and user B reconnects to user A via the IP interface. User A is completely unaware of this process.

\section{HANDOFF PROCEDURE}

The proposed handoff technique makes use of a network element which acts as SIP proxy server, SIP conference server and ISDN gateway. Whenever a call is made to user B or whenever a call

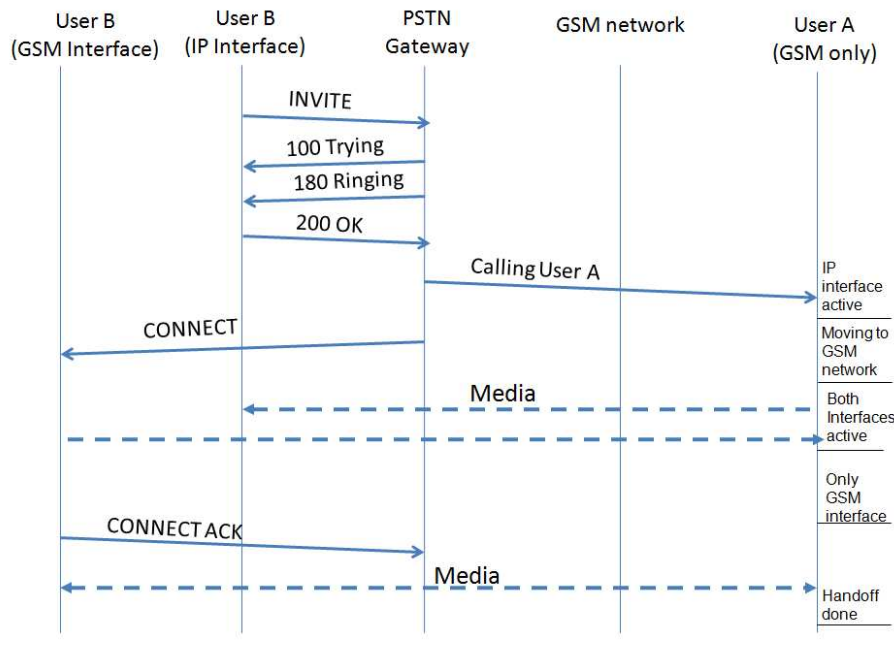

Figure 6: Handoff from the IP network to the GSM network when the user $B$ makes a call to user $A$

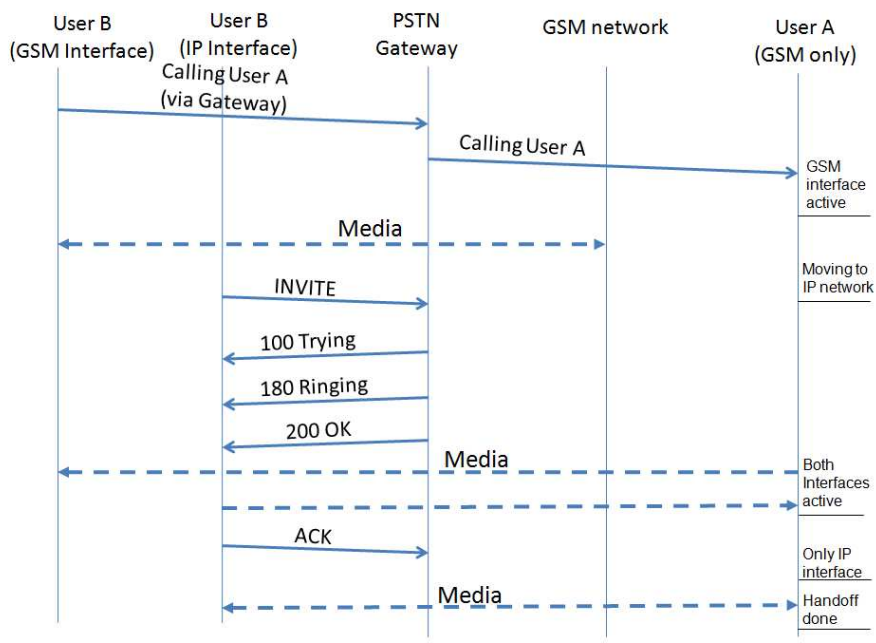

Figure 7: Handoff from the GSM network to the IP network when the user B makes a call to user $A$

is made by user B, the call is routed via the ISDN gateway. If user B makes a call through the IP interface, the call goes via the gateway which is also a SIP proxy. Again, if user B makes a call via the GSM interface, two-phase dialing occurs, first to the gateway and second to user A. So, in all the scenarios the call goes via the gateway. The handoff occurs whenever the user moves from an IP network to a GSM network or from a GSM network to an IP network. All the calls to and from the IP interface are SIP-based calls. The gateway gets the number of user B by the Redirecting number information element that is included in the packets sent to the gateway by the GSM network of user B. This number is vital for the implementation of our technique. Now let us describe in more detail the scenarios introduced earlier.

In scenario 1, when user A calls user B on the cellular interface, the call is forwarded by the cellular network of user B to the gateway. The gateway also acts as a SIP conference server. User $\mathrm{A}$ is put in a conference and waits for user B to be connected to the same conference. The conference is created dynamically with the caller-id of the incoming call used as the conference identifier. 
While user A is waiting in this conference, the gateway makes a call to user B. As explained previously, whenever a call gets forwarded, the diverted-from number (in this case user B's) is also sent to the diverted-to user (in this case the gateway). This information element contains the number of user B. After calling user $\mathrm{B}$ and adding it to the conference, both users are connected to the same conference and can start talking. When user B moves to an 802.11-covered region, handoff to the IP network occurs. Since such a handoff is a soft handoff, user B can connect to the same conference of its cellular interface using its IP interface and the conference ID of the existing conference with user A. For a small duration, all three interfaces, that is, one interface of user $\mathrm{A}$ and two interfaces of user B, are in the same conference. The cellular interface of user B is removed from the conference as soon as its IP interface has finished joining the conference.

Scenario 2 is very similar to scenario 1 ; the only difference is that the call is made to the IP interface of user B by the gateway. When a handoff to the cellular network occurs, the GSM interface of user B is added to the same conference as its IP interface and user A's interface. The IP interface is then removed once the GSM interface has joined the conference and connectivity between user B's GSM interface and user A has been established.

In scenario 3, user B makes a call via its IP interface. It uses SIP for establishing the call. Here the number to be called is present in the To header as part of the URI. The From URI contains the telephone number associated with user B. This is necessary because the From URI identifies the IP interface and the GSM interface of the handset. For example, the From URI of user B could be 1234567890@sipproxy.com, where 1234567890 is the telephone number of the GSM interface of user B. When user B moves out of the coverage area of the 802.11 network, handoff occurs. Here the handoff is from the 802.11 network to the cellular network. During handoff, the IP interface of user B is removed from the conference only after the GSM interface of user B has joined the conference. This is the conference in which user A's interface is already present.

In scenario 4, user B makes a call via the cellular interface. In this case, user B's call has to be routed via the gateway. The destination number is sent using Dual-Tone Multi-Frequency (DTMF) signaling. It is a two-phase dialing process where the call connects to the gateway first and then user A's number is dialed. For example, if user B wants to call 1234567890 which is user A's number and the gateway's number is $1-212-555-1234$, then the number to be dialed would be $12125551234 p 1234567890$. The user would have already added the gateway's number previously, while configuring the application, so that the only number user B would have to dial is user A's number. Here the handoff is similar to the previous scenarios where user B's cellular interface is removed from the conference as soon as user B's IP interface is added to such conference.

In all the four scenarios explained above, the handoff is initiated by user B. The handoff is automatically initiated by the client when there is a drop in 802.11 connectivity or when an 802.11 network becomes available.

In particular, for scenarios 2 and 3, when user B has to move from the IP network to the cellular network, it performs the handoff by calling with its cellular interface the gateway. At this point, the cellular interface of user B is added to the same conference of its IP interface. Once the cellular interface has joined such conference, the IP interface gets removed and the handoff can be considered complete.

Using a different approach, in scenarios 2 and 3, user B sends a REFER message to the gateway. When the gateway receives the REFER message, it makes a call back to the GSM interface of

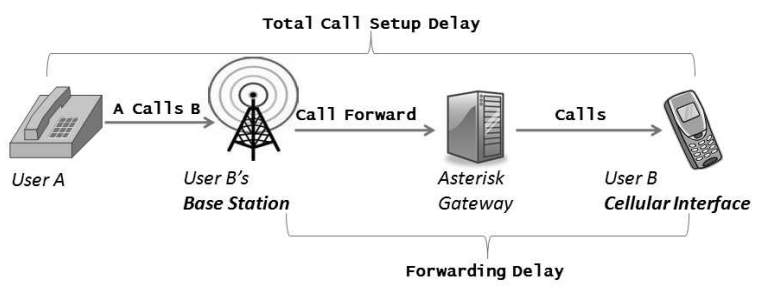

Figure 8: Delays introduced by CFB and Asterisk processing for a cellular-to-cellular interface call

user B and adds it to the same conference of user's B IP interface. As before, once the cellular interface has joined such conference, the IP interface gets removed and the handoff can be considered complete.

The call is terminated when either user A or user B leaves the conference.

\section{EXPERIMENTAL SETUP}

The experimental setup is shown in Fig. 1. To implement this technique, we modified the Asterisk open source PBX [4]. We used Asterisk as ISDN gateway, SIP proxy server and SIP conference server. X-Lite [13] was used as the SIP-based softphone. In particular, X-Lite was used as the IP interface of user B and a cell-phone was used as its corresponding GSM interface. The reason for this setup will be explained in Section 8 . We used a standard desktop machine with a $2 \mathrm{GHz}$ Intel Pentium $4 \mathrm{CPU}$ and $1 \mathrm{~GB}$ RAM as Asterisk server. Also, we used a Digium TE110P [14] card to connect Asterisk to a T1 trunk which is connected to the Columbia University PBX.

\subsection{Forwarding delay and total call-setup delay}

Forwarding delay is the delay introduced by call forwarding in the GSM network and the processing overhead in Asterisk. Total call-setup delay is the time measured from when user A presses the call button, to when user B receives an incoming call notification. Similarly, we have also measured this delay when user B calls user A. The time was measured using a stopwatch because of the absence of a GSM packet sniffer. In the following two sections we calculate these delays.

\subsubsection{Delay for a cellular-to-cellular call}

For a cellular-to-cellular call, user B gets a call notification on its GSM interface. The forwarding delay is shown in Fig. 8. This delay was calculated as an average over ten experiments and is approximately equal to the call setup time of two calls plus the extra time introduced by Asterisk. Forwarding delay from A to B is about $6.7 \mathrm{~s}$ whereas the total call-setup delay from A to B is $9.6 \mathrm{~s}$. Similarly, total call-setup delay from B to A is higher because of the DTMF digits being sent. Total call-setup delay is $15 \mathrm{~s}$ in our experiments.

\subsubsection{Delay for a cellular-to-IP call}

This delay for a cellular-to-IP call is similar to the delay for a cellular-to-cellular call. The difference is that, in the former case, the call is made to user B on its IP interface. In such case, the delay is less compared to the delay when the call is made to the cellular interface of user B. This is because of the higher bit-rates on an IP network. This delay is shown in Fig. 9. The forwarding delay from A to B was calculated as an average over ten experiments and is 


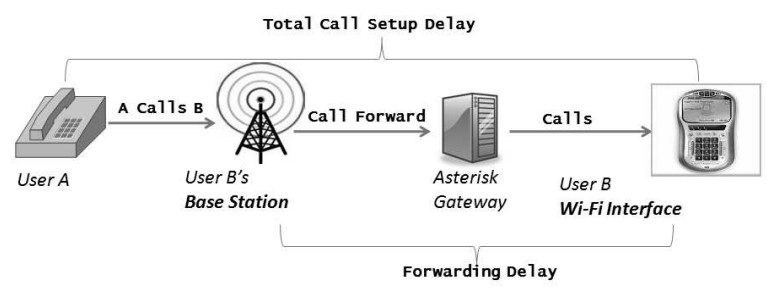

Figure 9: Delays introduced by CFB and Asterisk processing for a cellular-to-IP interface call

equal to $3.1 \mathrm{~s}$ while the total call-setup delay from A to B is equal to $6.2 \mathrm{~s}$. Similarly, the total call-setup delay from B to A is $6.9 \mathrm{~s}$.

\subsection{Handoff Delay}

We use a soft handoff technique where one interface is disconnected only after the other one is connected. This ensures that the user is always connected also during the handoff. The challenge here is to make sure that both local and remote users do not perceive that the call has been transferred from one interface to the other. The user should not feel a degradation in voice quality. We make use of ISDN layer 3 protocol called Q.931. The Q.931 protocol is responsible for setting up connections between the user station and the network and for terminating the connection when one of the parties in the connection issues a disconnect request such as going on-hook. We are mainly using two Q.931 message types to figure out when to complete the handoff. The two Q.931 packets we are using are CONNECT and DISCONNECT. The CONNECT message is used when the called party picks up the telephone. This message signals the acceptance of the call by the called party. The DISCONNECT message is sent when either the called or the calling party hangs up the telephone, that is, ends the call.

\subsubsection{Handoff Delay from ISDN to IP Interface}

For measuring the delay, Asterisk's source code (rtp.c) was modified so that it disconnects the ISDN trunk as soon as it gets the first RTP packet from the IP interface. The time was then calculated between receiving the first RTP packet from the IP interface and Asterisk sending the DISCONNECT message to the ISDN trunk. The timestamps for these messages were obtained from the debug log generated by Asterisk. The average delay, calculated over ten measurements, was $0.02 \mathrm{~s}$.

\subsubsection{Handoff Delay from IP to ISDN Interface}

For measuring the handoff delay when moving from an IP network to a cellular network, the Asterisk source code (q931.c) was modified so that the IP interface would get disconnected just after sending the CONNECT message but before sending the CONNECT ACK packet to the ISDN trunk. The time was then calculated between receiving the last RTP packet from the IP interface and before Asterisk sending the CONNECT ACK message to the ISDN trunk. Here again, the timestamp for these messages was obtained from the debug log generated by Asterisk. The average delay, calculated over ten measurements, was $0.1 \mathrm{~s}$.

\section{IMPLEMENTATION DETAILS}

In this section we describe briefly what a dialplan is and the main components of Asterisk we have used. Dialplan in Asterisk is the master plan of control or execution flow for all of its operations. It controls how incoming and outgoing calls are handled and routed. The behavior of all connections through the PBX is configured in the dialplan. MeetMe is the conference-server component of Asterisk. MeetMe has various features like the creation of dynamic conferences, adding and removing a user to and from a conference. MeetMe was extensively used for developing the proposed technique in Asterisk.

Fig. 10 shows a snippet of the dialplan that was written for our approach. This dialplan snippet gives a callback to the user whose call has been forwarded to Asterisk and then dynamically creates a conference. We maintain a database of all the users who are subscribers with a dual-mode handset and can, therefore, perform a vertical handoff. This is required to find if the call that has arrived to the gateway is from user B or user A. This is necessary because, as we have shown, calls from user A and user B are handled differently by Asterisk.

\section{IMPLEMENTATION CHALLENGES}

We encountered several challenges in the implementation of the proposed handoff technique. One of the major problems we faced was the fact that the gateway could not receive the telephone number of the diverted-from user. Although this number, as explained previously, is supposed to be sent to the diverted-to destination, it was not sent because of an older switch present in the Columbia University wired network which did not forward this information to our gateway. This forced us to hard-code the number statically in order to implement our approach in Asterisk.

We could not implement the handoff using the REFER message because of a bug in Asterisk. Any routing decisions for a call in Asterisk has to be done using the dialplan. The REFER message unfortunately does not go through the dialplan. This was also the case for the Contact header which we wanted to use in place of the To and From URIs being used in scenario 3.

For developing the application for a real handset, we faced the problem that open-source SIP clients for mobile devices were unavailable. Although open-source SIP based clients for Windows and Linux exist [15] [16], the lack of open source SIP-based clients for either Windows Mobile [17] or Symbian [18] made development of this technique on handsets difficult. Most of these projects have no support for the latest Windows Mobile platform or have outdated external libraries. For example, in the case of Minisip [19], there were various compatibility issues regarding the libraries that were being used. The $\mathrm{C}$ and $\mathrm{C}++$ libraries needed for Mobile 6 and present in Visual Studio 2005 are much more strict than the code which is written for Minisip. Therefore much of the Minisip source code is broken. Another open-source project we looked into was Pjsip [20]. This project has a good SIP stack but its user agent (UA) for the handset version is very limited.

We also faced problems with the APIs provided by Windows Mobile 6 [21]. After going through the Telephony APIs for Windows Mobile 6, we found out that such APIs were insufficient for implementing our technique. This is mainly because most of the dual-mode handsets available on the market have two sets of speakers, one which is exclusively used for GSM-related tasks and the other one which can be used by all other applications, including the SIP client. To better explain the situation, let us assume that a call is ongoing on the SIP client and now, using our technique we want to call using the GSM interface. In this case, the windows mobile handset will automatically mute the speaker for the SIP client and activate the speaker for the GSM interface. Unfortunately, the telephone speaker gets activated and the other speaker gets muted as soon as the dialing of the numbers starts. The other speaker remains muted until the call on the GSM interface is completed. There was no API able to control the telephone speaker. Also, this part of the handset is very dependent on the OEM's audio drivers 
Calls a phone number

exten => _.,14,system (cp /var/spool/asterisk/temp.call /var/spool/asterisk/outgoing/)

;Creates a conference dynamically

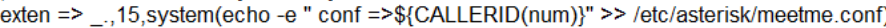

Puts the user in a conference

exten $=>$ _.,16,MeetMe $(\$\{$ CALLERID(num) $\} \mid 1 M q)$

\section{Figure 10: Asterisk dialplan}

and on how the sound gets routed to which speaker.

Our implementation, which is done on Asterisk, is a proof of concept to show that the proposed technique is feasible. Although we did manage to get Pjsip running on Windows Mobile 6 and wrote a very simple UA for it, complete implementation on the dual mode handset was not possible due to the various problems mentioned above.

\section{CONCLUSION}

We have proposed a novel mechanism to enable handoffs between IP and cellular networks by using a network element acting as an ISDN gateway, proxy SIP server and SIP conference server. Our design requires no changes to the existing network architecture and protocols, it only requires some off-the-shelf equipment.

Another advantage of the proposed approach is that it requires only a single telephone number for the ISDN gateway for all users. A different approach would have been to assign one telephone number per each user. This second approach would have the advantage of reducing the various delays mentioned earlier since the need for forwarding the call would be eliminated. In particular, user A would be directly calling the unique number assigned to user B. It would, however, introduce the obvious disadvantage of having one extra telephone number for every possible user.

\section{Acknowledgments}

This work was supported by the National Science Foundation under grant CNS 0202063, Sprint and FirstHand Technologies.

\section{REFERENCES}

[1] A. Shneyderman and A. Casati, "Fixed Mobile Convergence." McGraw-Hill Osborne Media, 2008.

[2] "Voice Call Continuity (VCC) between Circuit Switched (CS) and IP Multimedia Subsystem (IMS); Stage 2." 3GPP TS 23.206, 2007.

[3] R. Shacham, H. Schulzrinne, S. Thakolsri, and W. Kellerer, "Session Initiation Protocol (SIP) Session Mobility." Internet Engineering Task Force, Internet-Draft (work in progress), 2007.

[4] "Asterisk the open source telephony platform.” [Online]. Available: http://www.asterisk.org/

[5] M. R. G. Azada, R. P. Ejzak, J. J. MacNamara, D. Sand, and R. Thompson, "Seamless mobility across IMS and legacy circuit networks." Bell Labs Technical Journal, 2006.

[6] N. Banerjee, A. Acharya, and S. Das, "Seamless SIP-based mobility for multimedia applications." Network, IEEE, 2006.

[7] Salsano, S. Veltri, L. Martiniello, and G. Polidoro, "Seamless vertical handover of VoIP calls based on SIP Session Border Controllers." ICC '06, IEEE, 2006.

[8] H. Tze, "Handoff between VoWLAN and cellular," Master's thesis, Department of Electrical and Computer Engineering, University of Toronto, 2004.
[9] Q. Wang, M. Abu-Rgheff, and A. Akram, "Design and evaluation of an integrated mobile IP and SIP framework for advanced handoff management." IEEE International Conference on Communications, 2004.

[10] "Integrated Services Digital Network (ISDN); Diversion supplementary services; Digital Subscriber Signalling System No. 7 One (DSS1); Part 1: Protocol specification." ETSI EN 300 207-1, 2001.

[11] "Digital cellular telecommunications system (Phase 2+); Mobile radio interface layer 3 specification." ETSI TS 100 940, 2003.

[12] "Digital cellular telecommunications system (phase 2+); call forwarding (CF) supplementary services; stage 3." ETSI EN 300 952, 1999.

[13] “X-lite." [Online]. Available: http://www.counterpath.com/xlitedownload.html

[14] "Digium Wildcard TE110P Single T1/E1 PCI card VoIP SIP IAX Asterisk PBX.” [Online]. Available: http://www.voipsupply.com/

[15] "OpenWengo Freedom to Call, Freedom to Code." [Online]. Available: http://www.openwengo.org/

[16] “Columbia SIP user agent.” [Online]. Available: http://www1.cs.columbia.edu/ xiaotaow/sipc/

[17] "Windows mobile." [Online]. Available: http://www.microsoft.com/windowsmobile/default.mspx

[18] "Symbian OS.” [Online]. Available: http://www.symbian.com/

[19] "Minisip." [Online]. Available: http://www.minisip.org/

[20] "Open source embedded SIP stack and media stack from pjsip.org." [Online]. Available: http://www.pjsip.org/

[21] "Windows mobile 6." [Online]. Available: http://msdn2.microsoft.com/en-us/library/bb158486.aspx 